\title{
P 045 INTERNATIONAL PALLIATIVE CARE JOURNAL CLUB ON TWITTER: EXPERIENCE SO FAR
}

T Whitburn, ${ }^{1}$ C Walshe, ${ }^{2}$ KE Sleeman'. ${ }^{1}$ King's College London, Cicely Saunders Institute, Dept. of Palliative care, Policy and Rehabilitation, Bessemer Road, London SE5 9PJ; ${ }^{2}$ International Observatory on End of Life Care, Division of Health Research, Lancaster University, Lancaster LA1 4YG

10.1136/bmjspcare-2014-000838.48

Introduction@hpmJC (hospice and palliative medicine Journal Club, \#hpmJC) was launched in February 2014 on the social networking service Twitter, as a regular international journal club for palliative care. The journal club aims to encourage critical analysis of research methods and findings, and to promote evidence based practice, by providing a forum to discuss latest research findings.

Aim(s) and method(s) To analyse the use and reach of \#hpmJC, from the first journal club in February 2014, to date.

All data on Twitter posts (tweets) using \#hpmJC were extracted from Twitter using analytic tools Sysomos and Symplur. Outcomes included number of tweets, number of unique users, users' designated country, and impressions (potential number of accounts reached).

Results 7 journal clubs have taken place. 2360 tweets were sent, from 230 individual Twitter accounts and with contributions from people in 17 countries. For contributors whose country of origin is known (59\%), most were based in the UK $(41 \%)$ or USA $(26 \%)$. Tweets from resource-poor countries were initially uncommon but increased over the time period. The mean number of contributors at each journal club was 32. The potential reach of \#hpmJC varied, but for the most recent journal club was 290,802 unique users.

Conclusion(s) Social media provides opportunities to share expertise and disseminate information globally, transcending geographical boundaries.

$@$ hpmJC has been used to start a viable and sustainable online multidisciplinary journal club with wider geographical spread and potential reach than a traditional journal club. Strategies to increase participation in resource-poor countries are being developed. 